\title{
UNA HERRAMIENTA TIC ESTRATÉGICA PARA EL CRECIMIENTO PROFESIONAL EN LA SOCIEDAD DEL CONOCIMIENTO: \\ La formación transversal curricular de competencias comunicativas
}

\author{
MSc. María Caridad Valdés Rodríguez; mvaldes@uci.cu \\ MSc. Noralvis de Armas Rodríguez; noralbis@uci.cu \\ MSc. Susana Beatriz Darin; sbdarin@yahoo.com.ar \\ MSc. Margarita Abreu León; qumarqui@yahoo.es \\ Ing. Ariel Castro Arevalo \\ Universidad de las Ciencias Informáticas (UCI) - (UAl) - (UPR) \\ Ciudad de La Habana.
}

\section{RESUMEN}

En el análisis curricular del profesional sobre las competencias comunicativas solo se tiene como antecedente formativo explícito, en algunos programas de asignaturas, determinados objetivos, pero en los años y en las disciplinas no existe explícitamente. Es por ello que, a pesar de las acciones desde el curso de nivelación creado de Comunicación Profesional se ha concebido una herramienta estratégica para el crecimiento profesional en la sociedad del conocimiento: el eje transversal curricular de competencias comunicativas. Se fundamenta la producción en la práctica profesional productiva durante la formación comunicativa universitaria del ingeniero en Ciencias Informáticas. Se elaboraron varios portales didácticos, cursos en al Entorno Virtual de Aprendizajes. Se facilita la producción desde el sistema de actividades prácticas individuales y colectivas y de la evaluación un banco de materiales para el entorno de desarrollo de nuevos medios didácticos y de otras aplicaciones informáticas.

PALABRAS CLAVE: herramienta TIC, crecimiento profesional, sociedad del conocimiento, formación, competencias comunicativas

\section{ABSTRACT}

In the curriculum analysis of the professional on communicative competence, only certain objectives are considered as explicit antecedents for a few subject programs; therefore, a strategic tool for the professional growth in today's knowledge society has been developed: the curricular global issue of communicative competence. Production is based on the productive professional practice during the 
university communicative education of the engineer in Computer Sciences. Some didactic websites and courses in a Virtual Learning Environment have been created. Production is facilitated from the system of practical individual and group activities and from the evaluation of a bank of materials for the learning environment of new didactic means and other computer science applications.

KEY WORDS: ICT tools, professional growth, knowledge society, education, communicative competence.

\section{INTRODUCCIÓN}

Productividad de la comunicación en la era de internet.

Dr. Abraham Nosnik.

El presente trabajo surge ante la necesidad de perfeccionar en la formación del profesional la comunicación empleada en los diferentes procesos productivos dado que en las diferentes pruebas de calidad y en las auditorías correspondientes no pueden existir problemas comunicativos ni en los productos ni en las documentaciones. Varios levantamientos de conjunto con el área de calidad de la infraestructura productiva constatan la urgencia del perfeccionamiento en este tema.

En el análisis curricular del profesional sobre las habilidades comunicativas orales y escritas se tiene un antecedente formativo explícito en algunos programas de asignaturas determinados objetivos dirigidos a tal propósito, pero no en todos los años y disciplinas existe una concepción curricular que forme estas competencias con conciencia.

Es por ello que, a pesar de las acciones desde el curso de nivelación con la asignatura creada a tales efectos de Comunicación Profesional y las desarrolladas desde la estrategia de Lengua Materna, se ha concebido didácticamente para su implementación una herramienta estratégica para el crecimiento profesional en la sociedad del conocimiento: el eje transversal curricular de competencias comunicativas.

Es propósito en este trabajo fundamentar la herramienta diseñada desde la concepción curricular y su implementación práctica durante la formación comunicativa universitaria del ingeniero en Ciencias Informáticas.

Las TIC introducidas en la labor educativa hacen necesario el replanteamiento de las prácticas de enseñanza por parte de los profesores. Si bien la reflexión pedagógica asociada a la educación virtual hace especial hincapié en el alumno como centro del proceso, no se puede desestimar la importancia que en la enseñanza adquiere el docente en la enseñanza que conduce al aprendizaje más amplio e indicadores que se tienen en cuenta para buscar la excelencia en la labor docente: como la enseñanza flexible, el aprendizaje abierto, las competencias, los roles y tareas del docente. El trabajo didáctico con la herramienta producida para alcanzar tales fines contempla los nuevos escenarios y sus demandas para la Educación Superior, la situación de la docencia tradicional, el nuevo rol del docente universitario para satisfacer las necesidades actuales de formación y la comparación entre el rol tradicional del profesor y las nuevas características en la sociedad del aprendizaje. 
Durante los años de vida el ser humano va asimilando, enriqueciendo, modificando y conformando una educación personal, sus propios comportamientos, valores y principios todo lo cual en sus ámbitos laborales le facilitan la eficiencia, eficacia y calidad de vida social y profesional. Desde su formación tiende a potenciar el desarrollo profesional, lo cual lo encamina a su perfeccionamiento laboral y ciudadano.

Dentro de ese marco curricular institucionalizado la educación profesional o las prácticas profesionales mediante las acciones formativas y ejes transversales curriculares lo preparan y cualifican para la vida presente y futura de su profesión, desde integración de tareas y actividades docentes, investigativas y productivas, convirtiéndolos en hombre y mujeres más independientes, con mayor calidad en las trasferencias de conocimientos, aptitudes y habilidades en función de objetivos definidos durante sus actividades comunicativas de los servicios y los procesos productivos y con una alta satisfacción de sus productos y relaciones personales y laborales.

La diversa bibliografía consultada registra que ha sido interés durante todos los tiempos y en el presente de resolver los problemas que aún subsisten de cómo facilitar el crecimiento humano de estudiantes durante su formación profesional y de los trabajadores en las instituciones y empresas dado que la era de la oralidad convirtió al ágora en espacio de encuentro y aprendizaje, la de la escritura construyó y valoró a la biblioteca como espacio de memoria y saber y la era digital ha elevado a las redes a espacio de interacción y formación. El éxito de la escuela depende de la habilidad para hacer que esa mera presencia de artefactos tecnológicos se transforme en una integración a través del currículo.

Desde finales del siglo XX y principios del XXI en medio de los desafíos y retos de la sociedad del conocimiento, cuando se establecen estándares internacionales y se produce la ruptura de las barreras de la comunicación a través de "nuevas", las universidades se hallan ante un reto ineludible: adoptar estándares de calidad educativa internacionales para poder ser competitivos, sin abandonar sus propios estándares de calidad desarrollados a lo largo de años de experiencia académica. La globalización de la Educación Superior lleva a las instituciones educacionales a concretar acciones para hacerle frente a los desafíos que esta implica.

Se centra esta experiencia de diseño e implementación curricular en identificar y fundamentar una herramienta estratégica para el crecimiento profesional en la sociedad del conocimiento: la formación transversal curricular de competencias comunicativas desde la asignatura inicial de Comunicación Profesional, pasando por las que se potencian desde las diversas áreas del currículo, asignaturas, disciplinas y prácticas pre profesionales hasta las competencias necesarias para la eficiencia en los diferentes roles a formar durante cada año de la carrera.

Los autores se basaron en el presupuesto teórico de que la comunicación es un comportamiento concreto, una actitud que se puede adquirir con formación y que permite aprender, crecer, emprender y crecer profesional e integralmente a nuestros estudiantes como seres humanos, quienes están llamados a trasformar la sociedad del conocimiento.

Este tema de las competencias comunicativas se incorpora al pensamiento científico en la década de los sesenta del siglo XX, signado por la autoría lingüística de Noam Chomsky, quien la definió como "capacidades y disposiciones para la interpretación y la actuación". Esta definición se ha enriquecido hasta la actualidad no solo por lingüistas como él, sino también por profesionales de diversas áreas de la ciencia como la Psicología, la Pedagogía, la Sociología entre otras. Entre los más representativos que aportan a la evolución del concepto están: Dell Hymes, Canale y Swain, Caridad Cancio, Cot, Charaudeau, 


\section{Beltrán, la cubana Angelina Roméu Escobar, Hernández y Matos, Emilio Ortiz y Forgas, Parra y Mas, D.}

Zaldívar, Abraham Nosnik y Ackoff.

Ante tales fines y derivado de conocimientos y experiencias formativas se presenta en este trabajo una propuesta que ya tiene sus pasos en el perfeccionamiento como en la carrera de Ciencias Informáticas, con la anuencia de especialistas, productores y las opiniones de los alumnos en formación y egresados.

\section{DESARROLLO.}

La propia crítica apunta a que el desarrollo de las habilidades comunicativas, a pesar de ser en esencia un proceso activo, no ha escapado en la práctica de su enseñanza - aprendizaje al formalismo. Se alude a que esto ocurre sobre todo cuando se ha querido desarrollar dichas habilidades en situaciones desligadas de las necesidades práctico comunicativas, del estudiante, sin analizar que las actividades comunicativas que son el centro del esqueleto u organización conceptual de la enseñanza comunicativa del lenguaje, involucran el aprendizaje en comunicación auténtica y que además esto puede tener lugar en un contexto de interacción entre dos personas en una de las infinitas situaciones de la vida diaria; pero que también puede ser organizada para aprender fines, propósitos en el contexto de la clase u otros espacios educativos.

Una exigencia actual mundial y nacional es que la enseñanza debe aspirar a cambios cualitativos en las estructuras y sistemas de conocimientos, que irradien también cambios cuantitativos en el proceso. Específicamente la enseñanza de lenguas está llamada a instaurar el análisis de la competencia comunicativa, de los futuros egresados, vista como el desempeño comunicativo de los estudiantes al integrar las cuatro habilidades del lenguaje: hablar, escuchar, leer y escribir.

En la actualidad, la didáctica del habla, ha ocupado un lugar importante en el desarrollo de la enseñanza de la lengua. Esta didáctica está concentrada en el problema de la comunicación, es decir, en lograr que nuestros estudiantes sean capaces de comprender y comunicarse de forma coherente, en dependencia de las necesidades comunicativas que se les presenten ante diferentes situaciones, en que habrán de intercambiar.

En este sentido, se ha identificado la necesidad de formar un profesional que esté en capacidad de afrontar de una manera profesional el campo del conocimiento desde la perspectiva de los lenguajes informáticos y de actos productivos con base comunicativa, es decir, formar un profesional que sepa que, con las políticas de desarrollo económico y de apertura del país, las competencias son esenciales para los procesos de comunicación. Un profesional que pueda, entonces, proyectarse con mayor visión a fin de aportar al desarrollo integral del país en el marco de las exigencias internacionales. Se plantea que mejorando los procesos de comunicación, sube la confianza y credibilidad de la gente, base resultante para poder moldear el comportamiento deseado mediante formación concreta.

La complejidad que representa la temática del desarrollo de las competencias profesionales y dentro de ellas la comunicación, no solo abarca su concepción e instrumentación, sino que se extiende incluso a su formación. Al decir de las fuentes e investigadores del tema las competencias, no se producen espontáneamente ni de manera inmediata. Requieren de intencionalidad educativa, proporcionada por los sujetos que intervienen en el proceso (bilateralidad) y de una continua sistematización sino que la mayoría en el tiempo han coincidido en que se desarrollan en la acción, se construyen paulatinamente a medida que los alumnos se apropian de un conjunto de saberes. Involucran diferentes capacidades para el 
desempeño profesional y por lo tanto, suponen la puesta en juego de una escala de valores que las dota de sentido dentro de cada contexto específico

Dentro de ellas la competencia comunicativa ocupa un lugar importante. En la actualidad se reconoce como parte de las competencias profesionales. La necesidad de su desarrollo ha llevado su incorporación como exigencia de la Educación Superior dentro de la formación profesional de todos los estudiantes universitarios de los países.

En el contexto de las Ciencias Informáticas la comunicación informativa presupone un espacio productivo para la comunicación que se tiende y extiende a través de la comunicación vía digital: con mecanismos como la internet, el ser humano ha desplegado su universo, permitiendo que se expresen todas las identidades, es decir, los imaginarios sociales, donde el mensaje es materia prima del comunicador y los materiales con los que la mente configura el mensaje, son estímulos, significados, imágenes y valores.

Para basar los conocimientos en resultados, poder convertirlos en experiencia demostrable y corroborar que una competencia en auge es la gestión del tiempo y no muchas horas de trabajo sino una jornada inteligente

se ha basado la experiencia en una concepción metodológica de trabajo curricular con las competencias ha clasificado en dos grandes grupos a las mismas. Las denominadas concepciones estratégicas comprenden la visión y capacidad de conocer la comunicación en la comercialización, las relaciones comunicativas que se deben generar en los procesos del trabajo en equipo, en el alcance rápido, eficaz y económico la gestión de los recursos, cómo satisfacer al cliente, la cualidad empática al relacionarse con el cliente. Las denominadas competencias intratégicas abarcan las habilidades comunicativas en los procesos directivos, para liderar equipos, para ser emprendedor, optimista y autocrítico

La formación de profesionales competentes y comprometidos con el desarrollo social constituye hoy día una de las misiones esenciales de la Educación Superior. La sociedad demanda de profesionales capaces no solo de desenvolverse eficientemente en la solución de problemas de la práctica profesional, sino también de lograr un desempeño profesional con calidad, parece paradójico que el desarrollo tecnológico obligue al profesional de la comunicación a desarrollar competencias del lenguaje.

En la contemporaneidad se precisa de un profesionales con una sólida formación general, adaptable, flexible, capaz de conducirse con facilidad en las situaciones comunicativas originadas a partir de la asunción de los diversos roles que emanan de su perfil laboral; para lo cual requiere del desarrollo de la competencia comunicativa y denotar competitividad mediante adecuados comportamientos en actos comunicativos diversos.

Esta situación nos es ajena, pues el proceso de enseñanza - aprendizaje de las competencias del ingeniero en ciencias informáticas aún no posee un adecuado grado de sistematización que permita su rápida y eficaz identificación. Sin embargo, la propia práctica ha demostrado que la competencia comunicativa ocupa un espacio importante dentro de ellas, incluso puede considerarse como un eje transversal de la carrera.

En este sentido, el trabajo de la transversalidad de la comunicación en todas las áreas del currículo convierte a esta competencia en aprendizaje clave y puerta básica para acceder a múltiples conocimientos en otros campos, y como factor para la convivencia y las relaciones interpersonales en los procesos comunicativos personales, académicos y productivos. Les resulta entonces difícil cuando ya los colectivos pedagógicos y estudiantiles están permeados de estos presupuestos ignorar la formación de habilidades 
comunicativas, debido esencialmente a la estrecha relación existente entre las habilidades y las competencias.

Así el eje transversal curricular define el núcleo metodológico de las actividades del docente, como facilitador, orientador y educador en el proceso de enseñanza - aprendizaje, tiene la misión de procurar que a partir de las actividades cotidianas que organice para los estudiantes desde sus asignaturas se motive al alumno a que aprenda con rapidez y desarrolle las competencias comunicativas asociadas a su actuación profesional.

El estudio práctico se sustenta en el reconocimiento de la competencia comunicativa como un eje transversal en el proceso formativo de los estudiantes y en las relaciones que existen entre su desarrollo y la calidad de los productos y servicios productivos en los que participan con aspiración de excelencia.

La solución a este problema implica satisfacer la necesidad del conocimiento teórico y práctico sobre los subsistemas verbal - no verbal de la comunicación y del desarrollo con calidad de los productos y servicios productivos, el manejo de las cuatro competencias que conforman el dominio integral de una lengua (comprensión del lenguaje hablado, expresión oral, expresión escrita y comprensión de textos). Se agrega a esta labor experimental lo que para el paradigma pragmático de la comunicación es el contexto, ambiente o sistema. El contexto es el referente del proceso comunicativo.

Resulta muy importante estimular en las personas la medición de su propia evolución, haciéndoles asumir su responsabilidad de crecimiento profesional y emocional en estos procesos. Pero también los docentes mantienen su observación detenidamente durante el proceso y las actividades formativas y corrigen si es preciso y ello supone el desarrollo de otra manera de aprender, conociendo y haciendo uso de la comunicación por la vía también de los medios audiovisuales, debido al tipo de lenguaje que los caracterizan y que estimulan capacidades para globalizar, sintetizar, establecer relaciones y procesar informaciones, entre otras.

La propuesta curricular coloca en el andar pedagógico herramientas para promover la formación de competencias comunicativas en alumnos que resultan más eficientes y eficaces en sus actividades dada la preparación teórica, técnica y práctica de los diversos tipos de comunicación en sus roles profesionales: se entrenan desde la comunicación familiar, pasando en el aspecto oral y escrito, por la técnica, científica, mediática e informática, cada una de las cuales contiene principios, estilos y maneras de permitir la profesionalidad durante su formación y una vez egresados.

Así también con esta experiencia se alcanza la comprensión de ideas principales de textos de diferentes naturalezas y estilos comunicativos de cualquiera de las asignaturas del Plan de estudios, expresarse con fluidez, relacionar dos informaciones distintas y, con la propia experiencia de vida, emitir juicios morales, debaten, usan diversas fuentes, elaboran creativamente y con independencia sus tareas y trabajos profesionales y pueden comunicarse con empatía y claridad, usar distintos códigos y lenguajes. La eficiencia, la motivación de equipos, la asertividad o la creatividad, entre otras, son competencias valoradas por las instituciones y empresas que en varias ocasiones se ha constatado que los profesionales o quienes encauzan la formación de ellos no conocen bien o no han tenido presente potenciar desde el currículo y la práctica pre-profesional.

Atendiendo a que uno de los propósitos no logrado del todo con la calidad necesaria en el proceso docente - educativo en la escuela es el desarrollo de una eficaz competencia comunicativa en los estudiantes la 
didáctica de la lengua hoy tiene entre sus propósitos priorizar el trabajo con este concepto y su aplicación en todos los componentes que rigen la enseñanza de la lengua materna.

Otro de los valores es el nivel de conciencia que toma el alumnado de la importancia de la comunicación como herramienta estratégica ya que desde su concepción se le hace partícipe y conocedor de la estrategia curricular que considerará durante su formación.

No solo se consideró la comunicación como contenido transdisciplinar sino que se tuvieron presente las relaciones comunicativas también ya que la universidad es un entramado organizativo complejo y comunicacional y que produce un producto: el conocimiento, con metodologías y dinámicas interdisciplinarias y transdisciplinarias.

El nuevo milenio exige nuevas formas de acceso al conocimiento y en particular a la actividad universitaria; y la comunicación está llamada a contribuir a la diversificación de formas de distribución del conocimiento y a la competencia comunicativa en el desempeño profesional, en la relación universidad-empresa y en las acciones extensionistas para difundir los resultados de la investigación científica, lo que demuestra la validez de abordar la reconceptualización de las tareas de la universidad: docencia, investigación y extensión en su vínculo con la comunicación. Esto posibilita una mirada comunicativa a la universidad que considere su función y su contexto y permita su conceptualización como complejo entramado de relaciones comunicativas y una aproximación a la problemática de la universidad latinoamericana en el marco del vínculo institución-organización- comunicación y sus efectos profesionales.

Existe una valoración positiva en la calidad de la enseñanza universitaria de la necesaria capacitación en comunicación profesional, con vistas a alcanzar niveles superiores de eficiencia de las producciones orales y escritas en todos los contextos formativos, roles productivo e investigativos y comercializadores en los que interactúan los estudiantes y directivos con otros profesionales en Cuba y en el extranjero..

Se diseñó por las autoras un nuevo programa docente: Comunicación Profesional, el cual está insertado y tiene acceso desde el portal del curso y en el Entorno Virtual de Aprendizaje y está basado en una materia necesaria para los profesionales en formación. El mismo ya se ha impartido en el CPT de Informática UCI CUJAE y ha permitido un pilotaje y perfeccionamiento del mismo con vistas al curso regular diurno desarrollado en la nivelación de primer año del presente curso escolar.

A partir de la implementación de las TIC en los escenarios formativos actuales, se elaboraron varios portales didácticos que integran artículos referidos a la comunicación profesional en general y particular, contiene webgrafía, bibliografía, galerías de imágenes, acceso al Entorno Virtual de Aprendizajes UCl y la intranet de la ciudad digital.

Se facilita la producción desde el sistema de actividades prácticas individuales y colectivas y de la evaluación un banco de materiales para el entorno de desarrollo de nuevos medios didácticos y de otras aplicaciones informáticas que pueden ser empleados en la multiplicación de esta propia experiencia o como modelo para nuevos diseños de medios en contextos pedagógicos típicos.

Como parte de un nuevo módulo del curso de Formación Pedagógica en adelanto y de la divulgación y entrenamiento a Alumnos Ayudantes se han generado nuevos productos informáticos para el trabajo metodológico y docente de disciplinas tales como: Ingeniería y Gestión de software, Sistemas Digitales, Programación, Matemática y Ciencias Sociales. 


\section{CONCLUSIONES Y RECOMENDACIONES}

1. A partir de la implementación de las TIC en los escenarios formativos actuales se elaboraron varios portales didácticos (anexos), así como los cursos diseñados en al Entorno Virtual de Aprendizajes $\mathrm{UCl}$ que tributan al desarrollo de las competencias comunicativas en espacios de la práctica profesional o durante los procesos productivos y docentes.

2. Se facilitó la producción desde el sistema de actividades prácticas individuales y colectivas y de la evaluación un banco de materiales para el entorno de desarrollo de nuevos medios didácticos y de otras aplicaciones informáticas que han sido empleados en el trabajo metodológico, docente y productivo de las facultades y en sus proyectos o como modelo para nuevos diseños de medios en contextos pedagógicos típicos.

3. Se aporta una propuesta de variantes curriculares para la carrera UCI (abrir) la cual cuenta ya con resultados de pilotaje favorables y de empleo por colectivos de profesores y de Alumnos Ayudantes en la sede y en las Facultades Regionales, a considerar en otras regiones universitarias.

4. Se recomienda la generalización de la propuesta a todos los grupos y el mantenimiento de los portales y la creación de nuevos concursos, foros, talleres y actividades prácticas productivas que contribuyan con la motivación cultural idiomática y a elevar la calidad en la comunicación oral y escrita de todos en los diferentes contextos en que el universitario actúa, socializa y produce.

\section{BIBLIOGRAFÍA:}

1. Araneo, Paula. El poder de la comunicación institucional II. Imagen. Buenos Aires, 2001.

2. Benavides, Juan.: "Dirección de comunicación empresaria". Barcelona Gestión 2000 y 2001

3. Carrascosa, José Luis.: "Información/Comunicación". Arcadia. Madrid 2003.

4. Cervera Fantoni, Angel Luis.: "Comunicación total". Esic, Madrid, 2004.

5. Costa, Joan: "La comunicación en acción: informe sobre la nueva cultura de gestión". Paidós, Barcelona, 2001

6. Darin Susana, María Caridad Valdés Rodríguez, Antonio Rey Roque La educación virtual en la Sociedad del Conocimiento. En 5to Simposio sobre sociedad de la información, Argentina, ago 2007.

7. Devers, Thomas; "Aprenda a Comunicarse Mejor - Expresión no verbal, actitudes y comportamientos", Editorial Grijalbo, Buenos Aires, Argentina, 1990.

8. Escotet, Miguel Angel (1993) Tendencias, misiones y políticas de la universidad. Mirando hacia el futuro. UCA, Nicaragua.

9. Estrategias de

comunicación Autor: Rafael Alberto Pérez. www.ariel.es

10. García del Portal, Jesús (1998) La universidad latinoamericana: cultura de integración e identidad cultural, en Revista Cubana de Educación Superior. Vol. XVIII. No.3. La Habana. Cuba.

11. Germán Caicedo Prado: Gobernar es Comunicar. Editorial USC (Cali - Colombia). 2004

12. LACASA, S, Antonio.: "Gestión de la Comunicación empresarial". Gestión 2000. Madrid, 2004.

13. Lozada Díaz, José Carlos (Coord.).: "Gestión de la comunicación en las organizaciones". Ariel, Barcelona, 2004 
14. Martínez Solana, Yolanda.: "La comunicación institucional: análisis de sus problemas y soluciones". Fragua, Madrid, 2004

15. Piñuel, José Luis; Lozano, Carlos Ensayo general sobre la comunicación Barcelona: Paidós, 2006

16. Prieto Castillo, Daniel (2000) Comunicación, Universidad y Desarrollo, Maestría en Planificación y Gestión de Procesos Comunicacionales, Centro de Comunicación Educativa La Crujía, Facultad de Periodismo y Comunicación Social de la Universidad Nacional de La Plata, Buenos Aires, Argentina.

17. Ribeiro, Lair; "La Comunicación Eficaz", Editorial Urano, Barcelona, España, 1996

18. Sanchís, José Luis.: "Comunicar con éxito: teoría y práctica de la Comunicación". Ediciones Gestión 2000. Madrid 2004

19. Selva Domínguez, José Manuel, Carmenate Portilla, Angélica, y Cabrera Monroy, Francisca (1998) Gestión del Conocimiento, una nueva perspectiva, en El Trabajo del Conocimiento, instrumento de gestión, Congreso Nacional de Acede, Empresa y Economía Institucional, Asociación Científica de Economía y Dirección de la Empresa, Universidad de las Palmas de Gran Canaria. http://www.fcee.ulpgc.es/Acede98/acade/mesa02/2 02c.htm http://www.fcee.ulpgc.es/Acede98/acade/mesa02/2 02c.htm

20. Scheinsonhn, Daniel A. (1993) Comunicación Estratégica, Ediciones Macchi, Buenos Aires, Argentina.

21. Sotelo Enríquez, C., Introducción a la Comunicación Institucional, Ariel Comunicación, Barcelona, 2001

22. Tristá Pérez, Boris (1998) El enfoque de paradigmas en las instituciones de educación superior en Revista Cubana de Educación Superior. Vol. XVIII.No.2. La Habana. Cuba.

23. (1998) El enfoque de paradigmas en las organizaciones y la gestión del cambio en Revista Cubana de Educación Superior. Vol. XVIII.No.1. La Habana. Cuba.

24. Trujillo Fernández, María Heidi (1998) La competencia comunicativa de los profesionales del siglo XXI en Revista UNICSUL. Año 3. No. 4. Agosto. Universidad del Cruceiro del Sur. Sao Paulo. Brasil.

25. Valdés Rodríguez, María Caridad, Alicia Senra Mujica, Antonio Rey Roque, Susana Darín. Las Competencias Pedagógicas en los Creativos Entornos Virtuales de Aprendizajes Universitarios. En Edutec. Revista Electrónica de Tecnología Educativa. No. 24 / Diciembre 2007. En Educación y futuro digital, enero 2008

http://www.azc.uam.mx/publicaciones/gestion/num14/doc09.htm http://www.azc.uam.mx/publicaciones/gestion/num14/doc09.htm

26. Verón, Eliseo (1998) Entre la epistemología y la comunicación, Revista Digital - CIC - Cuadernos de Información y Comunicación.

http://ww.ucm.es/info/per3/cic/index.htm http://ww.ucm.es/info/per3/cic/index.ht

27. Zubeldía, María Lourdes, Viandana, Claudia Alejandra y Arriaga, María Cristina; "Cultura y Comunicación. Factores Estratégicos para el Cambio", 13ำ Congreso Nacional de Profesionales en Ciencias Económicas - Área V, San Carlos de Bariloche, Argentina, 2000, página 435 y subsiguiente. 


\section{Anexo I:}

Producciones informáticas generadas para y derivadas de la experiencia con la formación transversal curricular de competencias comunicativas.
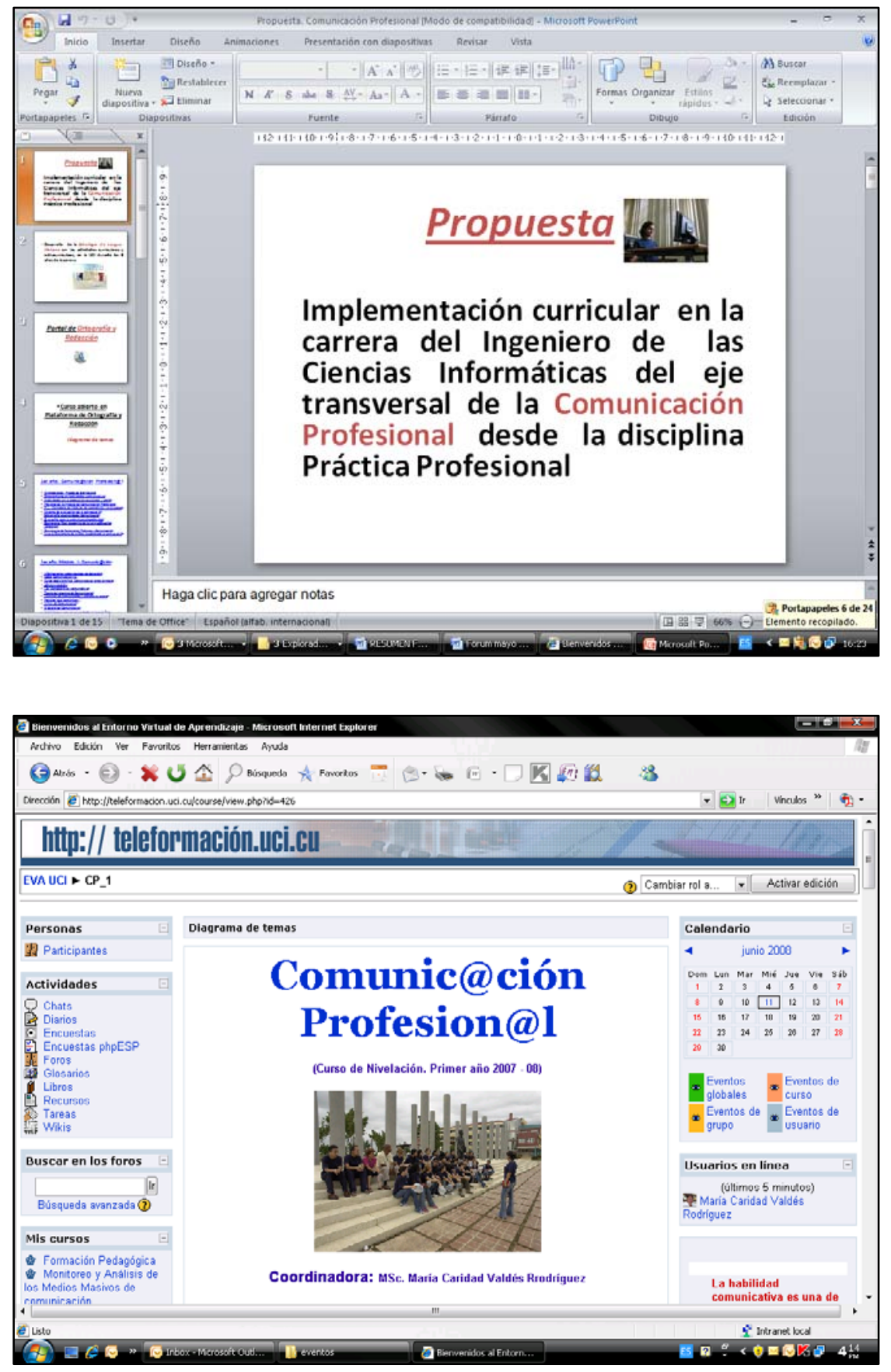

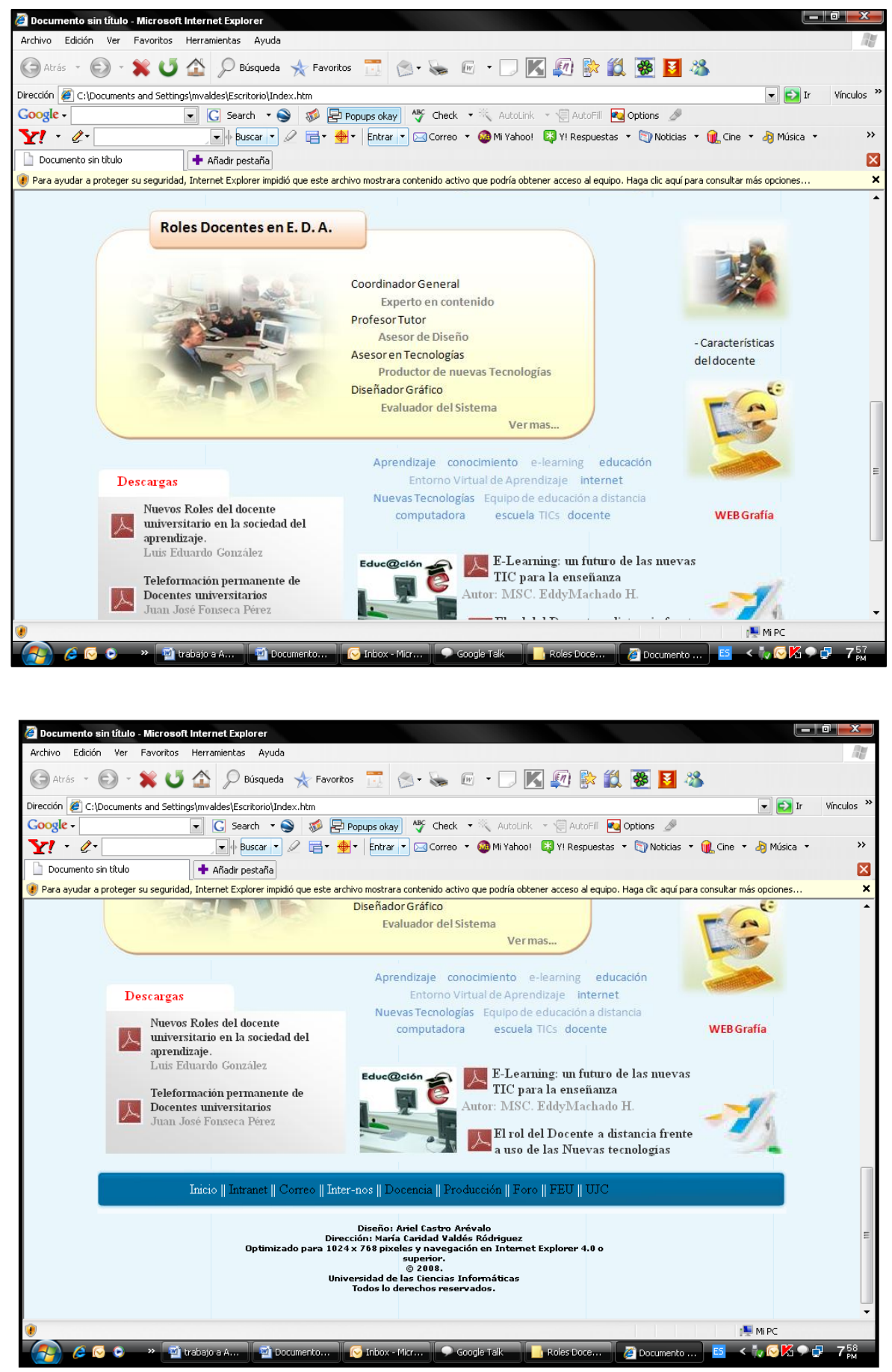

Para citar este artículo:

VALDÉS, M.C.; DE ARMAS, N.; DARIN, S.B.; ABREU, M.; CASTRO, A. (2008) «Una herramienta TIC estratégica para el crecimiento profesional en la sociedad del conocimiento: La formación transversal curricular de competencias comunicativas» [artículo en línea]. EDUTEC, Revista Electrónica de Tecnología Educativa. Núm. 26/Julio 2008. [Fecha de consulta: dd/mm/aa].

http://edutec.rediris.es/Revelec2/Revelec26/

ISSN 1135-9250. 